Culture et histoire dans l'espace roman

$6 \mid 2011$

Figures du pouvoir dans la littérature hispanoaméricaine

\title{
Trujillo-Balaguer ou les jeux de l'autorité et du pouvoir dans La Fiesta del Chivo
}

Claire Sourp

\section{CpenEdition}

Journals

Édition électronique

URL : https://journals.openedition.org/cher/9860

DOI : $10.4000 /$ cher. 9860

ISSN : 2803-5992

Éditeur

Presses universitaires de Strasbourg

Édition imprimée

Date de publication : 30 juin 2011

ISBN : 978-2-35410-033-9

ISSN : 1968-035X

Référence électronique

Claire Sourp, "Trujillo-Balaguer ou les jeux de l'autorité et du pouvoir dans La Fiesta del Chivo », reCHERches [En ligne], 6| 2011, mis en ligne le 17 décembre 2021, consulté le 27 janvier 2022. URL http://journals.openedition.org/cher/9860 ; DOI : https://doi.org/10.4000/cher.9860

\section{c) (i)(2)}

Ce(tte) œuvre est mise à disposition selon les termes de la Licence Creative Commons Attribution Pas d'Utilisation Commerciale - Partage dans les Mêmes Conditions 4.0 International. 


\title{
Trujillo-Balaguer ou les jeux de l'autorité et du pouvoir dans La Fiesta del Chivo
}

\author{
CLAIRE SOURP \\ Université Rennes 2
}

$I^{1}$ suffit de poser son regard sur la couverture du roman La Fiesta del chivo pour comprendre que le romancier péruvien Mario Vargas Llosa situe celui-ci d'emblée dans la thématique du pouvoir. L'illustration choisie est un des motifs de la fresque d'Ambrogio Lorenzetti: "L'allégorie du mauvais gouvernement». Le message ne peut être plus explicite lorsque le lecteur associe le personnage central du tableau, ce mauvais gouvernant cornu au bouc annoncé par le titre et lui-même offert en offrande au diable ${ }^{1}$, comme si un pacte avait été signé avec lui, justifiant par là même les horreurs commises par le régime en place. En effet, le bouc auquel se réfère le titre, c'est Rafael Leonidas Trujillo, personnage historique mis en scène ici par Vargas Llosa pour venir rappeler les heures sombres du régime dominicain, une figure du pouvoir dans l'espace textuel et en dehors de lui. Quand l'instrumentalisation du pouvoir passe par une répression féroce, dans la représentation fictionnelle de même que dans la réalité, le concept de pouvoir est accompagné de celui de violence, rappelant par là, au cœur même de la fiction ce que la philosophe allemande Hannah Arendt observait dans l'essai intitulé Du mensonge à la violence:

rien n'est plus fréquent que l'association du pouvoir et de la violence; il est extrêmement rare de les trouver séparés l'un de l'autre, sous leur forme pure

1 Un bouc se situe aux pieds du mauvais gouvernant, ce qui nous permet cette double lecture du terme bouc, en tant que maître du pouvoir - comme nous le laissent également entrevoir les vers du merengue qui servent d'épigraphe - et comme victime émissaire, un bouc également, mais à laquelle nous ne nous attachons pas dans ce travail. 
et donc extrême. Il n'en résulte pas cependant que l'autorité, le pouvoir et la violence ne soient qu'une seule et même chose (147).

Si l'on se réfère à la définition d'autorité pour venir compléter cette triade énoncée par Hannah Arendt, on peut lire: pouvoir d'imposer l'obéissance, organe du pouvoir, pouvoir de se faire obéir. De la même façon que violence et pouvoir sont intimement liés, lorsque autorité et pouvoir le sont aussi n'annoncent-ils pas une modalité nouvelle, celle de l'autorité poussée aux limites de l'autoritarisme en imposant l'obéissance par la force, de manière violente ? Autorité, pouvoir, violence semblent ainsi venir tisser leur toile autour des lecteurs pour les conduire tout au long de cette allégorie du $\mathrm{XX}^{e}$ siècle où le bouc prend des traits dominicains. Dans son analyse de l'institution de la polis, Myriam Revault d'Allones écrit: «Le pouvoirest déposé au centre afin qu'il ne soit la chose de personne, parce qu'il est la chose de tous» (49). Valable dans le cadre de la cité grecque qui fonctionne autour de l'agora et dans un régime démocratique qui confère le pouvoir au peuple, cette citation n'est pas applicable au cas de Trujillo. Le nom même de la capitale, Ciudad Trujillo, démontre que si le pouvoir est déposé au centre de la cité, il n'est pourtant la chose que d'un seul homme: le tyran. Il faut néanmoins tenir compte du caractère atypique de la situation dominicaine. En effet, il s'agit d'un régime bicéphale: d'un côté Trujillo, chef de gouvernement, et d'un autre Balaguer, chef de l'État. Pour Adolfo Castañón, il ne s'agit en fait que d'une apparence lorsqu'il affirme: «cabría pensar que Trujillo y Balaguer cumplen, en última instancia, una sola función con los dos rostros de un solo cuerpo: el poder» (196). Toutefois, notre analyse va s'attacher à distinguer les prérogatives de chacun des deux personnages car, selon la perspective adoptée, tous les deux n'ont pas la même relation au pouvoir. Le tableau ci-dessous va nous permettre de synthétiser ces données car, pour comprendre comment se répartissent le pouvoir et l'autorité entre les deux hommes d'État, il faut envisager trois perspectives: celle des Dominicains sympathisants du régime ou qui le subissent, celle des Dominicains qui s'opposent activement au régime et enfin, l'opinion des autres gouvernements, en particulier celui des ÉtatsUnis. Dans les trois cas, les compétences reconnues à Trujillo et à Balaguer ne sont pas les mêmes. Ce qui entre en jeu dans la confrontation de ces trois perspectives, c'est l'idée de légitimité. 


\begin{tabular}{|c|c|c|c|}
\hline & $\begin{array}{c}\text { Pour la majorité des } \\
\text { Dominicains }\end{array}$ & $\begin{array}{c}\text { Pour les Dominicains } \\
\text { opposants au régime }\end{array}$ & $\begin{array}{c}\text { Pour les gouvernants } \\
\text { étrangers (États-Unis) }\end{array}$ \\
\hline $\begin{array}{c}\text { TRUJILLO } \\
\text { Chef du gouvernement }\end{array}$ & Pouvoir ET Autorité & Pouvoir SANS autorité & Ni pouvoir ni autorité \\
\hline $\begin{array}{c}\text { BALAGUER } \\
\text { Président de la République }\end{array}$ & Ni autorité ni pouvoir & Autorité SANS pouvoir & Pouvoir ET autorité \\
\hline
\end{tabular}

\section{a. «El presidente fantoche $»^{2}$}

Avant tout, l'usage du titre et l'utilisation de la parole sont frappants dans le rapport qui unit Trujillo à Balaguer. Le président en titre, qui peut lui aussi prétendre à l'appellation "excelencia », l'utilise très respectueusement à l'égard de Trujillo. Ceci n'a pas échappé à la sagacité du dictateur qui lui fait remarquer "usted nunca me dice Jefe, como los demás» (288). Que déduire de l'emploi de tel titre plutôt que de tel autre? Flatterie? Volonté verbale du président fantoche de ne pas faire allégeance au chef du gouvernement dont juridiquement il est l'alter ego ? Volonté de la part de Trujillo de rappeler à l'ordre celui qu'il considère comme un vassal, de nier l'existence politique de celui qui n'a été pendant un temps que son ombre (287). Le mécanisme du pouvoir et de l'autorité se cristallise en premier lieu dans la parole et dans l'emploi des titres. Dès lors, le jeu du pouvoir, dans le rapport de force qu'il sous-tend, est palpable. Trujillo ne s'adresse jamais à son interlocuteur en le désignant par un titre honorifique distinguant son rôle et son pouvoir politique. Il appelle Balaguer « doctor », ce qui, malgré le grade le rabaisse dans l'échelle des valeurs puisque, comme le dictateur le lui rappelle cruellement, «Mi opinión sobre intelectuales y literatos siempre ha sido mala. En el escalafón por orden de méritos, en primer lugar los militares [...]. Literatos e intelectuales, los últimos. [...] Usted es una excepción, doctor Balaguer» (295). Trujillo affirme par là et malgré tout, de façon doublement négative et ironique, la piètre opinion qu'il a du Président.

L'antagonisme et le problème d'autorité et de légitimité entre les deux hommes est perceptible dans leurs propos et dans leur façon d'être, comme nous allons le voir à travers cet exemple:

el diligente poeta y jurista [Balaguer] que se había puesto de pie y se acercaba a saludarlo:

-Buenas tardes, Excelencia. [...]

2 Cette expression apparaît à plusieurs reprises dans le texte. Il n'est pas anodin qu'elle apparaisse également dans la bouche des conspirateurs qui reconnaissent par là que, de leur point de vue, Balaguer n'a aucun pouvoir individuel réel. 
- ¿Autorizó usted hace un par de semanas la salida al extranjero de la hija de Agustín Cabral? (282).

La courtoisie de Balaguer à l'encontre de Trujillo, est marquée dans le discours par le mot « excelencia » puis dans les gestes: Balaguer se lève pour aller à la rencontre de son visiteur et le saluer. Mais le dictateur ne répond pas à ces marques de politesse et, d'emblée, affirme sa supériorité face à un interlocuteur qu'il ne gratifie pas du moindre salut et qu'il apostrophe sans préalable sur le sujet de sa visite. Il est à remarquer que cet échange a lieu debout, et que, bien qu'il se trouve dans le bureau de Balaguer, contre toute attente, Trujillo se comporte en maître de céans et invite le Président à s'asseoir. Protocole surprenant. Il se retirera de l'entretien sans même dire au revoir.

Balaguer, malgré son titre de Président, n'a aucun pouvoir ${ }^{3}$, et avant de prendre une décision, il doit en référer à Trujillo, par le biais de «memorándum»(282). Il lui obéit donc, comme il le reconnaît: «seguí sus instrucciones» (285). Le nain du régime dominicain, celui qui n’a pas de pouvoir, doit rester une marionnette entre les mains du dictateur puisque toute initiative est ouvertement condamnée ${ }^{4}$. Par conséquent, sur simple injonction du généralissime, il doit obtempérer dans l'instant à ses désirs. La demande de signature de la promotion du favori en est l'exemple. Dans son raccourci, les mots renvoient au rôle de Balaguer qui ne détient qu'un pouvoir de façade exécutant les volontés du Benefactor. «Firme el ascenso [...] Así lo haré, excelencia» (305). Ainsi donc, la parole de Trujillo plie l'autre à son désir. Balaguer se soumet servilement à l'autorité et au pouvoir de Trujillo, son chef: «mi único vicio ha sido servirlo» (289), lui confesset-il. Quant à l'opinion que le dictateur a du président, elle est sans appel: "un hombrecito sin luz propia, como la luna, al que Trujillo, astro solar, iluminaba» (290).

C'est donc uniquement l'opinion internationale qui reconnaît à Joaquín Balaguer le cumul du pouvoir et de l'autorité. Tout d'abord parce que suite aux sanctions adoptées lors de la réunion de l'OEA, en 1960 à San José, «il n'y a plus dans l'empire de place pour la dictature dominicaine» (Lauro Capdevila: 209) et qu'un nouveau président de la République émerge.

3 C'est du moins l'image que l'auteur veut nous donner de lui en créant cette relation entre les deux hommes d'État. Le lecteur comprendra pourtant en fin de roman que Balaguer connaissait le projet de tyrannicide, «la complicidad de Balaguer tranquilizaría a los yanquis» (401).

4 La signature apposée sur l'autorisation permettant à Urania de quitter le pays et l'irritation qu'elle suscite chez Trujillo en sont la preuve. 
Les États étrangers ne sont pas dupes et comme le titrent les journaux de l'époque: "Rien de nouveau sur le front»(210). Pourtant, le roman présente le président comme le principal interlocuteur pour deux questions particulièrement épineuses: «La Iglesia, los Estados Unidos» (285). C’est lui qui est en pourparlers pour trouver un arrangement. Ces missions, celle menée auprès des États-Unis en particulier, expliquent donc que, pour l'opinion internationale, Balaguer représente à la fois l'autorité et le pouvoir.

Remarquons enfin que, après l'assassinat de Trujillo, Balaguer réussit à s'imposer dans le pays et, qu'en faisant preuve d'autorité, il s'empare du pouvoir. Sa charge factice devient réelle, de «presidente fantoche» (287) aux ordres de Trujillo, de «don nadie» (464), il est devenu «el auténtico Jefe del Estado» (idem) et il passe sur le devant de la scène (métaphoriquement représentée par les flashs des photographes, 493). Par conséquent pour asseoir son autorité et son pouvoir sur son peuple, il encourage d'abord l'exil de la famille Trujillo et instaure une loi d'amnistie politique. Ces deux mesures habiles visent à réconcilier l'opinion publique des Dominicains sympathisants et opposants de la dictature; elles jouent à la fois sur la continuité qui rassure les premiers et sur la rupture avec l'ancien régime qui, elle, satisfait les seconds.

\section{b. Trujillo, I'homme du pouvoir}

Le statut de Trujillo est également complexe, il s'impose dans le roman en tant que détenteur du pouvoir et de l'autorité. Il a d'abord mis en place un système de répression, le SIM, dans ce cas c'est la peur qui légitime l'autorité du tyran. Pour aussi paradoxal que cela puisse paraitre pour un dictateur dont les actes se réclament de la devise des tyrans - "Qu'ils me haïssent pourvu qu'ils me craignent $»^{5}-$, Trujillo a soin d'entretenir l'image paternelle de la bonté, en se faisant appeler Bienfaiteur, Père de la nation, protecteur... A ces images valorisées répondent alors les marques de dévouement et d'admiration d'hommes et de femmes du peuple et de courtisans. Ce processus de fidélisation suscite pourtant des interrogations perplexes chez Urania qui cherche à comprendre ce qui le motive et le justifie: «¿Qué les hacía? ¿Qué les daba, para convertir a don Froilán, a Chirinos, a Manuel Alfonso, a ti,

5 Oderint, dum metuant, expression du poète tragique Accius, citée par Cicéron et mise dans la bouche du tyran Attrée. Dictionnaire Le petit Larousse illustré, Paris, 2005, page XXIII. 
a todos sus brazos derechos e izquierdos, en trapos sucios?» (75). La jeune femme pose là une des questions fondamentales du roman qui oscille entre deux engrenages: celui qui conduit à l'assujettissement et celui qui mène à l'acte libérateur du tyrannicide. L'un comme l'autre constituent des attitudes antinomiques en réponse à l'autoritarisme du chef.

Pour les hommes de main de Trujillo et pour la plupart des hommes du peuple, le dictateur incarne à la fois le pouvoir et l'autorité. Trujillo assied son autorité sur l'intimidation, comme en atteste cette réflexion: "A mí no me tiembla la mano cuando tengo que matar - añadió después de una pausa-. Gobernar exige, a veces, mancharse de sangre. Por este país, he tenido que hacerlo muchas veces. Pero soy un hombre de honor» (119). Le personnage ne remet à aucun moment en cause les décisions qu'il a prises et que, selon lui, l'exercice du pouvoir justifie. Le verbe «exigir» situe l'action politique envisagée par Trujillo dans l'ordre de l'impératif catégorique auquel il ne peut se soustraire. C'est d'ailleurs parce qu'il agit, selon lui, conformément à la règle morale qu'il s'est imposée, qu'il continue à se considérer comme «un hombre de honor». Toutefois, l'usage que fait Trujillo de l'impératif catégorique qui fixe sa conduite est diamétralement opposé à l'exemplaire démarche éthique préconisée par Emmanuel Kant: "Agis de telle sorte que tu uses de l'humanité en ta personne comme en celle d'autrui, toujours comme une fin, jamais simplement comme un moyen» (41). Dans le système de Trujillo, le respect à la personne n'a pas de place, il ravale ses semblables en humanité à de la vermine:

Qué agradable era dar curso para la rabia cuando no había en ello riesgo para el Estado, cuando se podía dar su merecido a las ratas, sapos, hienas y serpientes. Las panzas de los tiburones eran testigos de que no se había privado de ese gusto (36).

Cette citation nous éclaire à double titre: tout d'abord, parce qu'elle conforte le lecteur dans cette idée qui va croissant que l'action répressive de Trujillo est, selon son point de vue, non pas abusive mais juste; d'autre part, parce que la formulation atteste de la perversité et surtout de l'abus de pouvoir du personnage: "ese gusto». La devise française d'Ancien Régime «car tel est mon bon plaisir» conviendrait parfaitement à la traduction de son corrélat dominicain pour traduire l'omnipotence du monarque et renvoie étroitement à la structure même de la tyrannie. Dans La République de Platon, après avoir défini quatre formes de régimes politiques, Socrate dit de la tyrannie: «Il est [des citoyens] que [le tyran] soupçonne de nourrir des idées de liberté qui les conduisent à refuser son autorité. Il trouve alors les 
moyens et l'occasion de les faire disparaître en les livrant à l'ennemi» (392). L'autorité du tyran se fonde donc sur un rapport de force qui lui est toujours favorable et il fait de la violence un instrument de pouvoir. Si nous pensons à la théorie établie à partir des exactions nazies dans laquelle Hannah Arendt démontre que, dans la relation d'obéissance à un ordre, le mal se banalise, nous pouvons envisager le personnage de Trujillo comme celui qui définit le sens alors que ses hommes de mains, le mettent en œuvre. Pour la philosophe allemande, cette violence bureaucratique est «insupportable car elle dissocie l'acteur et le sens ou la portée de ses actes ${ }^{6}$. Dans cette perspective, il semble que la violence soit la ligne directrice de la politique de Trujillo qui instaure la menace de la répression comme moyen de pression sur son peuple. Dans ce cas, il n'y a donc pas d'utilisation de la violence légitime d'État, une forme de violence fondée sur la rationalisation des conflits et la connaissance des limites à ne pas dépasser. Le sens donné par Trujillo se fonde sur la terreur par le recours à la violence systématique et illégale et rend compte de la cruauté du personnage. C'est cette image qui parcourt le roman, entretenue par des attitudes, des réflexions ou des ordres, comme ceux exécutés par Johnny Abbes: «Los echamos a los tiburones ayer mismo. Vivos, como usted mandó» (37). C'est d'ailleurs le supplice que le tyran pense infliger aux évêques états-uniens qui lui montrent quelque résistance. Pour Trujillo donc, la torture est la manière la plus radicale d'imposer son pouvoir. Les trente et un ans de règne ont banalisé le mal et celui-ci devient le principal mode d'action politique employé par Trujillo pour diriger le pays. Peu importe la barbarie de l'acte, dans ces nouveaux jeux du cirque sans spectateurs, le potentat fait jeter des hommes à la mer, peu après dévorés, en quelques secondes, par des requins, le pouvoir devient ainsi abus de pouvoir. Différents événements apparaissent donc comme des situations où la violence devient un instrument de domination, là où seule l'autorité du chef devrait s'affirmer. En prenant conscience que l'autorité de Trujillo est infondée, les personnages entrent en dissidence et participent à la conspiration. Tout d'abord Amador García Guerrero qui, pour prouver sa loyauté envers Trujillo, a dû tuer un homme et apprend, après le meurtre, qu'il s'agissait du frère de son amie, un communiste. Puis c'est Antonio de la Maza qui souhaite venger la mort de son frère et tue «el asesino de Galíndez, de Murphy, de Tavito, de las Mirabal, de miles de dominicanos» (122). Antonio Imbert, autre conspirateur, reconnaît lui aussi le pouvoir de Trujillo

6 Cette théorie énoncée par Hannah Arendt dans Eichmann à Jérusalem, Rapport sur la banalité du mal est reprise et analysée par Michel Wieviorka (246). 
sans croire toutefois à son autorité. Il n'a pourtant d'autre choix que de s'y soumettre, comme il convient à la perversité des régimes autoritaires:

Había sido ese malestar de tantos años, pensar una cosa y hacer a diario algo que la contradecía, lo que lo llevó, siempre en el secreto de su mente, a sentenciar a muerte a Trujillo, a convencerse de que, mientras viviera, él y muchísimos dominicanos estarían condenados a esa horrible desazón y desagrado de sí mismos, a mentirse a cada instante y engañar a todos, a ser dos en uno, una mentira pública y una privada prohibida de expresarse (187).

Cette citation fait la lumière sur le rapport qu'entretiennent la majorité des Dominicains (représentés dans la première colonne de notre tableau) à l'autorité. Il ne s'agit chez eux que d'une reconnaissance feinte qui provoque un "malestar» dû̀ à une forme de schizophrénie entre la sphère publique et privée. La citation joue donc sur le double, et la duplicité s'impose à qui veut éviter la dénonciation. Antonio Imbert révèle que la plupart de ses concitoyens jouent sur l'ambivalence et ne se soumettent qu'en apparence à la poigne du dictateur ${ }^{7}$, à son pouvoir car ils savent que dès qu'une voix dissonante se fait entendre, elle est écartée ${ }^{8}$. C'est parce qu'en Trujillo autorité, pouvoir et violence ne font qu'un, comme s'il était l'incarnation du vocable allemand qui envisage les trois dans une même logique: gewalt ; qu'un contre-pouvoir se met en œuvre pour tenter de rétablir l'ordre mis à mal par la dictature. Dans le cas des conspirateurs et, au-delà d'eux, des opposants au régime, Trujillo personnifie le pouvoir, sans incarner l'autorité. Le tyrannicide est alors la seule issue pour rétablir la relation nécessaire entre les deux, pour que l'autorité redevienne «la propriété ou l'attribut d'un pouvoir légitime, [...] ce supplément ou ce surcroît qui légitime le pouvoir» (Revault D’Allones 2006: 23).

Figure du pouvoir, incarnation de l'autorité dans son assimilation à l'autoritarisme, Trujillo s'insinue dans toutes les strates ${ }^{9}$ pour y insuffler une

7 Le cheminement de Salvador Estrella Sadhalá est un peu différent de celui des autres conspirateurs car pour lui, croyant fervent, Trujillo n'est que le représentant de l'autorité temporelle.

8 Cette réalité touche aussi bien les Dominicains dont l'idéologie n'est pas en accord avec le régime que les proches de Trujillo, comme Agustín Cabral. La liste des disparitions dont le dictateur est l'instigateur est fournie, mais incomplète: José Almoina, Jesús de Galíndez, les trois sœurs Mirabal, Bayardo Cipriota, les hommes et les femmes qui ont participé au complot du 14 de Junio, Tavito de la Maza... La main du dictateur règle les comptes, y compris en dehors du territoire national. C'est le cas de José Almoina qui est tué pour avoir publié, en Argentine et au Guatemala (85), deux livres contre Trujillo ou de Jesús de Galíndez qui se trouvait à Manhattan la dernière fois qu'il a été vu vivant.

9 Cette figure du pouvoir n'est pas sans rappeler la structure qu'Hannah Arendt étudie comme structure de l'oignon qui s'applique au tyran autoritaire. 
violence d'état illégitime: torture, viols, disgrâces, chaque Dominicain peut être affecté par cette instrumentalisation du pouvoir que Trujillo met en place à travers ses hommes de main, les hommes politiques et autres calies qui sillonnent la ville à la recherche des opposants. Figure complexe qui concentre tous les pouvoirs alors même que Balaguer n'apparaît aux yeux de tous que comme une coquille vide qui attend son heure pour récupérer le pouvoir et l'autorité dont il est spolié par les actes de Tujillo ${ }^{10}$. Toutefois, alors que tous pensaient que sans tête, le régime s'effondrerait de lui-même et que le peuple les acclamerait au lieu de leur tourner le dos, les conspirateurs se rendent eux-mêmes compte que tout ne se déroule pas comme prévu: «Fue como si se abriera la tierra, como si desde ese abismo, se levantara riéndose de él la carcajada del Maligno» (251). Il n’y a pas eu d'arrêt de la violence, et leur sacrifice (seul l'un d'entre eux échappe à la mort) aura été vain puisqu'il instaurera finalement un «trujillismo sin Trujillo» (Frauke Gewecke 2001: 109), comme si, y compris post mortem, le personnage continuait à concentrer les attributs du pouvoir et de l'autorité.

\section{Bibliographie}

Arendt, Hannah, 1972, "Qu'est ce que l'autorité?», in La crise de la culture, Paris, Gallimard, Folio essais.

_, 1966, Eichmann à Jérusalem, Rapport sur la banalité du mal, Paris Gallimard.

_, 2003, Du mensonge à la violence, essais de politique contemporaine, Paris, CalmanLévy, Agora.

Capdevila, Lauro, 1998, La dictature de Trujillo, Paris, L'Harmattan, Horizons Amérique latine.

Castañón, Adolfo, 2001, «La última fiesta del faraón», Revista Mexicana del Caribe, $6^{e}$ année, 12, Universidad Autónoma de Quintana Roo Chetumal, p. 189-200.

Dictionnaire Le petit Larousse illustré, 2005, Paris, page XXIII.

Gewecke, Frauke, 2001, «Presentación », Madrid-Frankfurt, Iberoamericana, 3.

Kant, Emmanuel, Fondement de la métaphysique des mœurs, http://classiques.uqac. ca/, p. 41.

Platon, 1995, La République, Paris, Le Livre de Poche, Classiques de la philosophie.

Revault D'Allones, Myriam, 2006, Le pouvoir des commencements, essai sur l'autorité, Paris, Seuil, La couleur des idées.

Vargas Llosa, Mario, 2000, La Fiesta del Chivo, Madrid, Alfaguara.

10 «Sondeó al Presidente fantoche, preguntándole si, en caso de desaparición súbita de Trujillo, "colaboraría con los patriotas". Su respuesta fue críptica: "según la Constitución, si Trujillo desapareciera, se tendría que contar conmigo"» (401). 
Wieviorka, Michel, 2004, La violence, Paris, Éditions Balland, Voix et Regard, p. 246. 